\title{
Erratum
}

\section{Argos induces programmed cell death in the developing Drosophila eye by inhibition of the Ras pathway}

\author{
Kazunobu Sawamoto, Akiko Taguchi, Yuki Hirota, Chiharu \\ Yamada, Ming-hao Jin and Hideyuki Okano
}

Cell Death and Differentiation (1998) 5, 262-270

The publishers wish to apologise for the misrepresentation of Figure 5 published in the above paper.

The correct version of Figure 5 appears below.
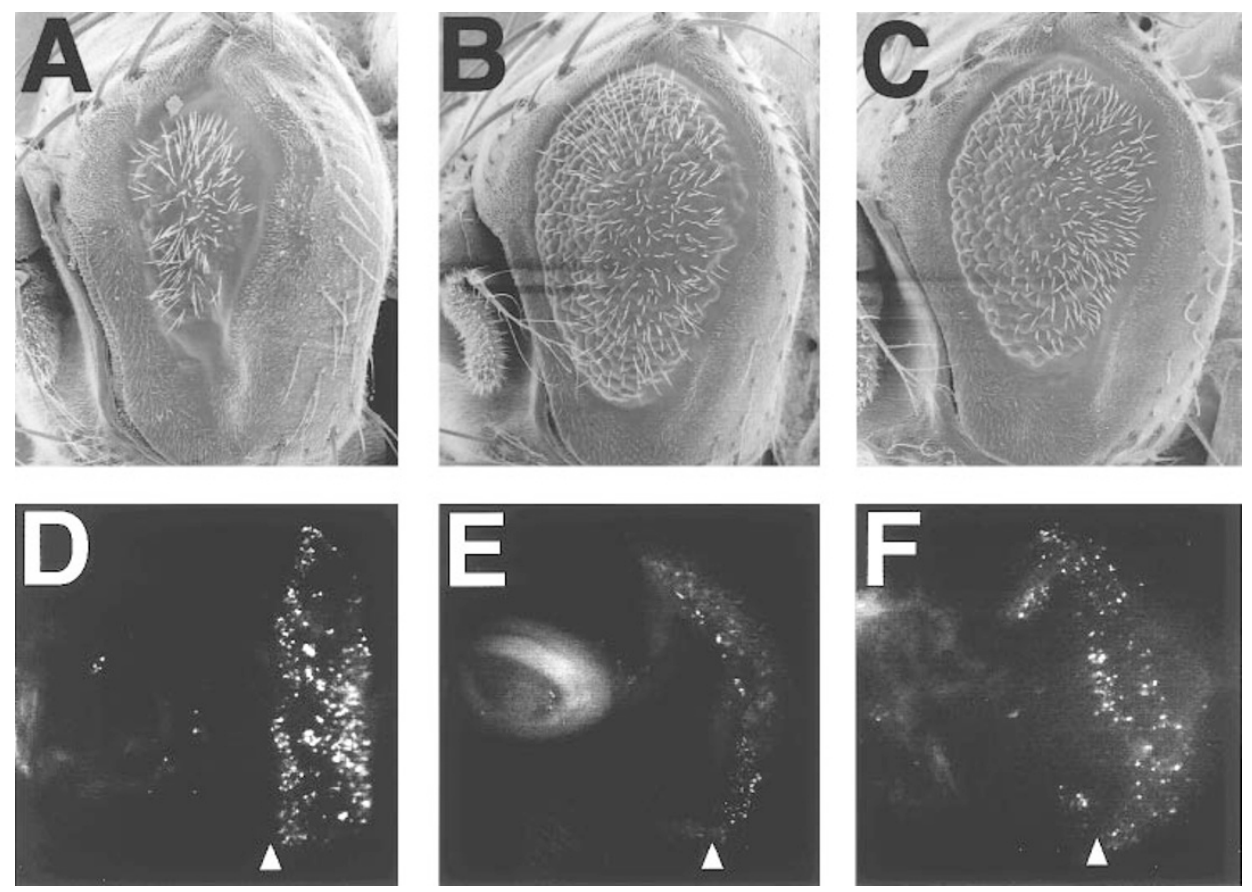

Figure 5 Hyper-activated MEK and MAPK signaling suppress Hid-induced cell death. (A-C) Scanning electron micrographs of compound eyes. (A) GMR-hid/+.

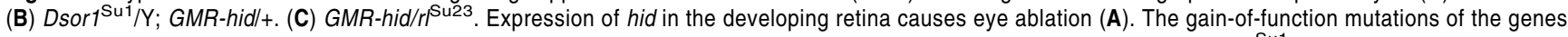
encoding for MEK (B) and ERK (C) restore the ommatidia. (D-F) Acridine orange staining of eye discs from GMR-hid/+(D), Dsor1 1 su1/Y; GMR-hid/+(E), and GMRhid/ri ${ }^{\beta u 23}(\mathbf{F})$ larvae. Anterior is to the left. Arrows indicate the position of the morphogenetic furrow. High levels of excessive cell death are present posterior to the morphogenetic furrow in the GMR-hid/+eye discs (D). The excessive cell death induced by Hid was significantly suppressed by the mutations, Dsor ${ }^{\text {Su1 }}$ (E) and $r l^{\mathrm{Su} 23}(\mathrm{~F})$ 